\title{
Bringing to Life a Newly Discovered Signature of Fatigue Crack Initiation via Multimodal Characterizations
}

\section{Dalton Shadle}

Cornell University

\section{Kelly Nygren}

Cornell High Energy Synchrotron Source

Jean Stinville

University of Illinois

\section{Marie Charpagne}

University of Illinois

\section{Timothy Long}

Johns Hopkins University

\section{McLean Echlin}

University California Santa Barbara https://orcid.org/0000-0003-4107-0366

\section{Christopher Budrow}

Budrow Consulting

\section{Andrew Polonsky}

https://orcid.org/0000-0003-4515-9827

\section{Tresa Pollock}

Materials Department, University of California, Santa Barbara https://orcid.org/0000-0001-9948-1083

Matthew Miller ( $\nabla$ mpm4@cornell.edu )

Cornell University https://orcid.org/0000-0002-0241-769X

\section{Article}

\section{Keywords:}

Posted Date: January 6th, 2022

DOI: https://doi.org/10.21203/rs.3.rs-1189770/v1

License: (1) (1) This work is licensed under a Creative Commons Attribution 4.0 International License.

Read Full License 


\title{
Bringing to Life a Newly Discovered Signature of Fatigue Crack Initiation via Multimodal Characterizations
}

\author{
D.J. Shadle ${ }^{1}$, K.E. Nygren ${ }^{1,2}$, J.C. Stinville ${ }^{3}$, M.A. Charpagne ${ }^{3}$, T.J.H. Long ${ }^{4}$, M.P. \\ Echlin $^{5}$, C.J. Budrow ${ }^{6}$, A.T. Polonsky ${ }^{5}$, T.M. Pollock ${ }^{5}$, and M.P. Miller ${ }^{1,2}$ \\ ${ }^{1}$ Sibley School of Mechanical and Aerospace Engineering, Cornell University, Ithaca, NY, USA \\ ${ }^{2}$ Cornell High Energy Synchrotron Source, Ithaca, NY, USA \\ ${ }^{3}$ Department of Materials Science and Engineering, University of Illinois at Urbana-Champaign, Urbana, IL, USA \\ ${ }^{4}$ Department of Materials Science and Engineering, Cornell University, Ithaca, NY, USA \\ ${ }^{5}$ University of California Santa Barbara, Santa Barbara, CA, USA \\ ${ }^{6}$ Budrow Consulting, Ithaca, NY, USA
}

December 2021

\begin{abstract}
Fatigue is the most prevalent failure mode in structural materials, yet remains challenging to study due to the seemingly unpredictable nature of crack initiation. To elucidate the driving forces of crack initiation in ductile polycrystalline metals, we employ a multimodal approach to identify and track grains with a suspected potential to initiate fatigue cracks via a newly founded signature. We discover this crack initiation potential (CIP) signature under the hypothesis that slip localization, a well-known precursor to crack initiation, is linked to intragrain misorientation, which can be quantified through single grain orientation distributions. We verify the CIP signature in an Inconel-718 material via static two-dimensional and three-dimensional electron microscopy and "bring to life" the dynamics of the CIP signature via in-situ synchrotron X-ray diffraction. With this CIP signature, we move to better focus studies of fatigue crack initiation on the individual grains and processes that drive fatigue failure.
\end{abstract}




\section{Introduction}

With over a century of study, fatigue failure is still the most prevalent failure mode in engineering components with 50-90\% of structural failures resulting from cyclic loading induced fatigue [1-4]. To combat this problem, engineers often rely on managing fatigue with methods like safe-life replacement and infinite-life design, which can be inefficient and dangerous if applied under the wrong assumptions [5]. To truly conquer fatigue, one must have a fundamental understanding of the microscopic processes that drive fatigue. In general, fatigue failure is commonly separated into three sequential steps: fatigue crack initiation, slow crack growth, and fast crack growth to final failure. Depending on the material and loading conditions, fatigue crack initiation can account for the longest portion of a component's life, yet it is the most difficult step to study due to the inability to predict locations where fatigue cracks may initiate. Even though there must be deterministic processes driving fatigue at some size scale, fatigue crack initiation appears to be entirely stochastic. One way forward is to have the ability to "watch everywhere, at all times" to discover a microscopic crack as it initiates to understand the evolving microstructural and micromechanical features that drive fatigue. This paper presents the discovery of a signature capable of tracking the fatigue crack initiation potential within a material.

One of the most common classes of structural materials susceptible to fatigue crack initiation is polycrystalline metallic alloys. A ductile alloy is comprised of self-contained units called crystals (or grains) that have atoms arranged in orderly patterns. Under cyclic loading conditions, common in fatigue failures, deformation can localize along these patterns of atoms in the form of crystallographic slip and can lead to crack initiation within a grain. Specifically in nickel-based superalloys, prior studies by Villechaise et al and Larrouy et al theorize that areas where localized slip impinges on a neighboring grain boundary, but the slip cannot transmit into the neighboring grain, are favorable crack initiation sites due to the slip shearing the physical grain boundary [6,7]. A recent high-resolution deformation study by Stinville el al on the Inconel-718 nickel-based superalloy furthered these findings by coupling two-dimensional electron backscatter diffraction (2D-EBSD), an microscopic orientation field measurement, and heaviside digital image correlation (H-DIC), a microscopic strain field measurement, to spatially correlate the same slip impingement behavior with plumes of intense intragrain lattice rotation (up to $10^{\circ}$ ), termed microvolumes, as shown in Figure 1 [6-11]. These observations, when combined with the crack initiation theory above, provide microvolumes as measurable features that may indirectly identify grains with the most potential for initiating a fatigue crack. The seemingly impossible problem of finding an unpredictable microscopic crack as it initiates somewhere in a metal becomes

a more tractable goal with identification of the grains with high crack initiation potential (CIP), narrowing the search from the microscopic crack-scale to the grain-scale. 

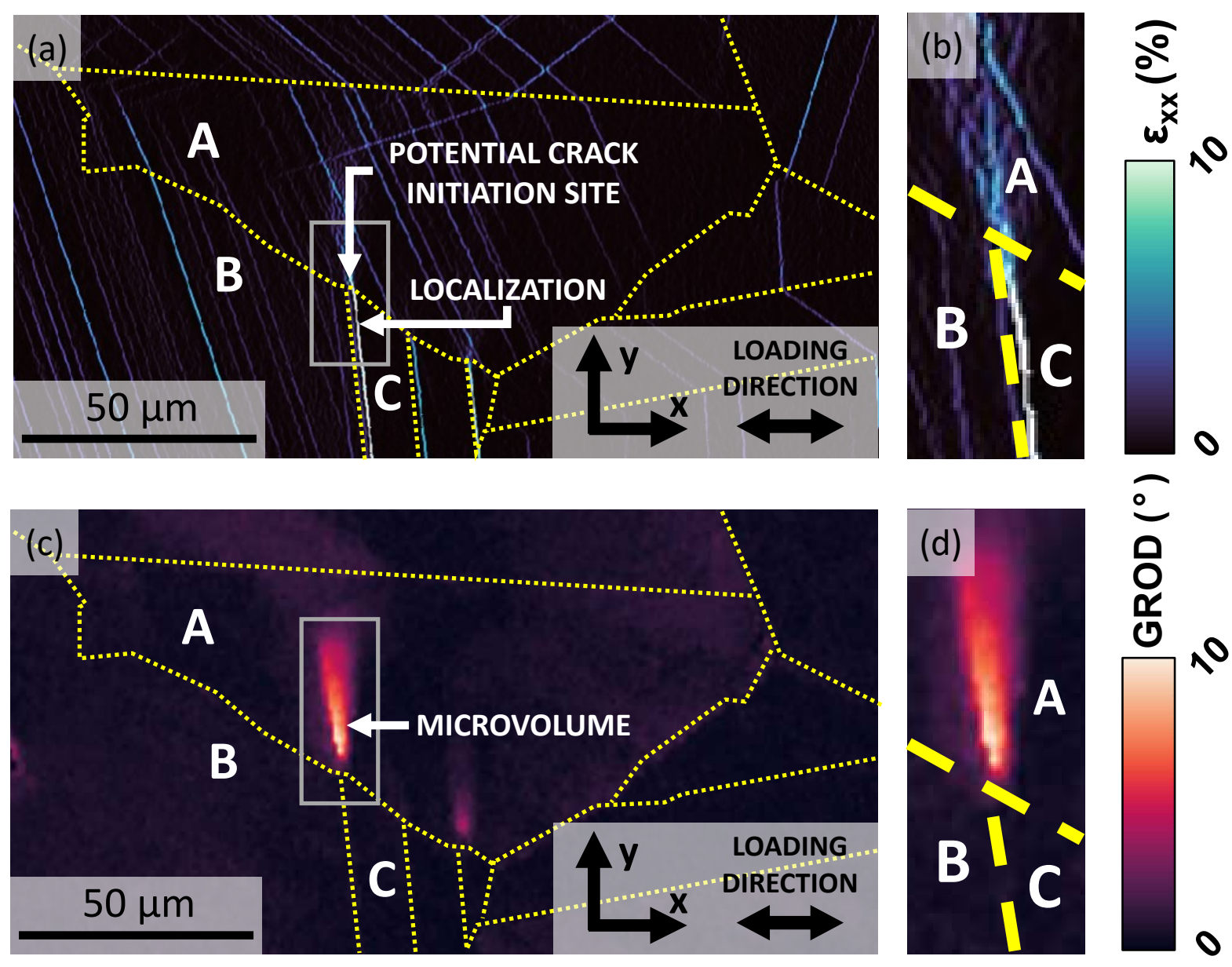

Figure 1: (a) H-DIC surface strain field of a Inconel-718 material, deformed in tension to a macroscopic loading direction strain of $1.26 \%$, depicting a grain neighborhood with grain boundaries outlined in yellow. A slip localization event in Grain $\mathrm{C}$ is labeled. A potential crack initiation site is labeled where the slip localization intersects the grain boundary between Grain A and Grain C. (b) A region of interest, indicated by a grey box in (a), where the slip localization in Grain C intersects the grain boundary of Grain A. (c) 2D-EBSD misorientation field of the same neighborhood colored by grain reference orientation deviation (GROD), a metric for measuring spatial differences in orientation based on the current grain-averaged orientation [12], with the grain boundaries outlined in yellow. The area with large gradients of GROD is termed a microvolume in Grain A near the intersection of the slip localization in Grain C is labeled. (d) A similar region of interest, indicated by the grey box in (c), depicting a microvolume in Grain A caused by the impingement of slip localization in Grain C. The crack initiation theory of Villechaise et al and Larrouy et al suggests that a crack has the favorable potential to initiate at the intersection of slip impingement of Grain $\mathrm{C}$ and microvolume of Grain A $[6,7]$.

Microvolumes are commonly identified through empirical observations of static 2D-EBSD surface scans. The complexity of dynamic in-situ measurements of bulk material (material containing many hundreds or thousands of surface and subsurface grains) necessary for "watching everywhere, at all times", prompts a more objective and perhaps statistical identification technique for grains with high CIP. To achieve this goal, we rely on a multimodal approach that combines both static and dynamic characterization techniques 
integrated through the commonality of a signature of CIP. A signature is a distinguishing measurement that characterizes a target phenomenon of interest primarily used to anticipate future events [13]. A common CIP signature would enable the statically measured microvolume to be figuratively brought to life through the time-resolved information provided by in-situ measurements.

In another recent study, the authors theorized that intragrain lattice rotation is quantified through approximations of single grain orientation distributions termed discrete single grain orientation distributions (DSGODs) [14]. A DSGOD is a weighted list of non-spatially-resolved orientations that exist within one grain and describes the orientation field density of that grain. Though a DSGOD cannot reconstruct the spatial mapping of an orientation field, we hypothesize that the higher order statistical moments of a DSGOD can serve as a common signature of CIP by quantifying the distinct lattice rotation of grains containing a microvolume. A schematic depicting the CIP signature is presented in Figure 2. The microvolume in Grain A shown in Figure 2(a) manifests as a skewed orientation distribution with a long tail shown in Figure 2(b). Grains with less intragrain misorientation, like Grain D shown in Figure 2(c), have smaller, more uniform orientation distributions as shown in Figure 2(d). The CIP signature is defined as the statistics that quantify DSGODs in search for these skewed, long-tailed distributions of grains containing microvolumes, which are linked to fatigue crack initiation. 

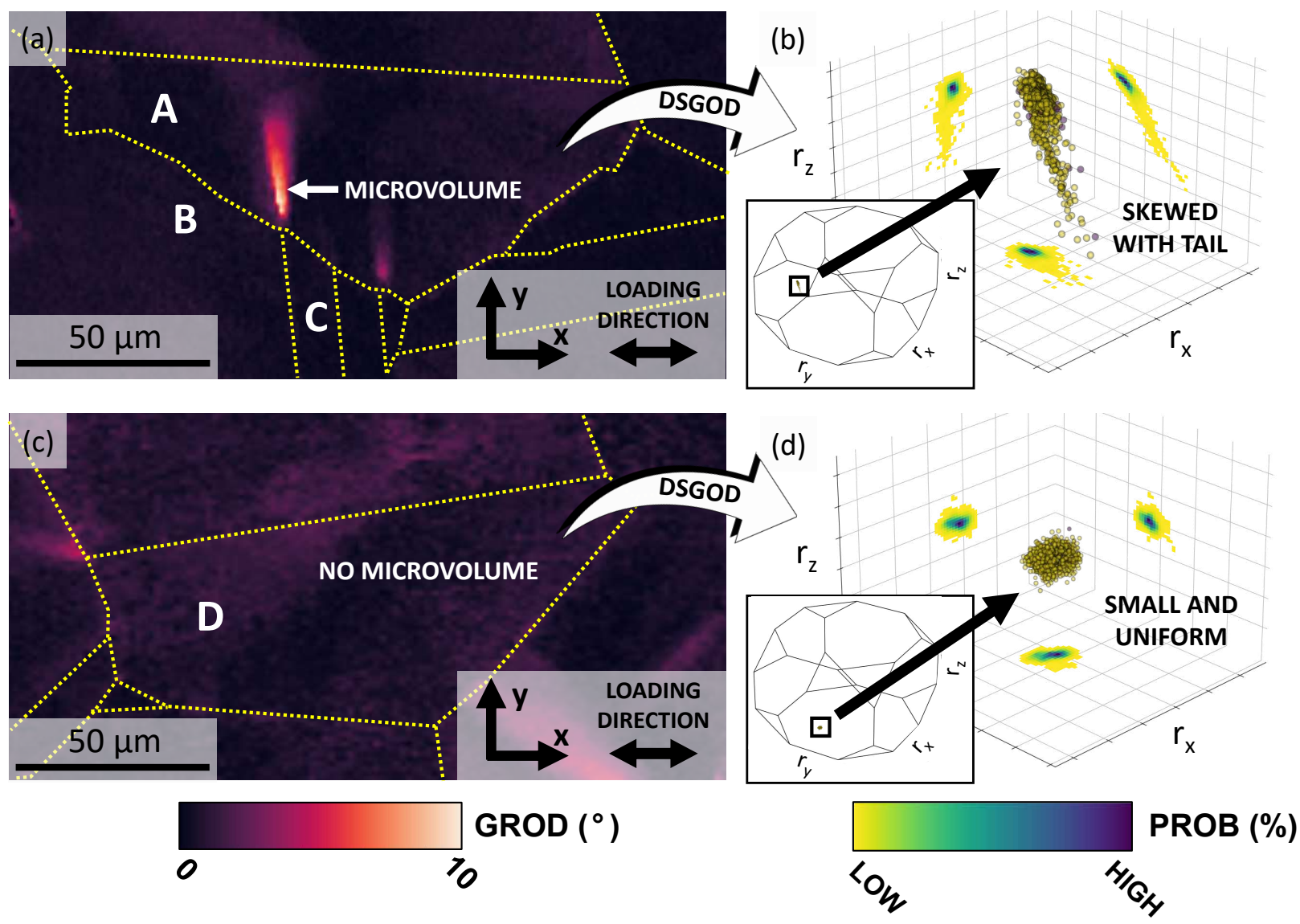

Figure 2: Mapping DSGODs to microvolumes as the crack initiation potential (CIP) signature we present in this study. (a) 2D-EBSD misorientation field of the same grain neighborhood of Figure 1 colored by grain reference orientation deviation (GROD) with the grain boundaries outlined in yellow. Grain A contains a microvolume as indicated by the arrow. (b) The discrete single grain orientation distribution (DSGOD) of Grain A depicted in a magnified portion of the Rodrigues parameterization of orientation space with the 2D projected orientation densities plotted on the far faces of the plot area. An inset of the cubic fundamental region of orientation space with the DSGOD location of Grain A is provided for context. (c) 2D-EBSD misorientation field of another grain neighborhood colored by GROD with the grain boundaries outlined in yellow. Grain D does not contain a microvolume. (d) The DSGOD of Grain D depicted in a magnified portion of orientation space with the $2 \mathrm{D}$ projected orientation densities plotted on the far faces of the plot area. An inset of the DSGOD location of Grain D in the fundamental region is provided.

Having defined the CIP signature, techniques for measuring, validating, and tracking the signature are necessary. 2D-EBSD and H-DIC measurements supply the necessary information to measure and validate DSGOD statistics as the CIP signature on the surface of the material. Furthermore, EBSD measurements can be extended into the material's subsurface to measure and validate the CIP signature via TriBeam measurements [15]. The TriBeam technique collects spatially-resolved three-dimensional (3D) measurements of grain orientations via femtosecond laser-enabled serial sectioning with EBSD data collected at every slice. However, these electron characterization techniques are not capable of studying fatigue crack initiation in-situ and subsurface simultaneously. For this condition, synchrotron-based far-field high energy X-ray diffraction 
microscopy (ff-HEDM) is the choice tool for non-destructively characterizing 3D volumes of polycrystalline material under in-situ conditions like cyclic loading [16-23]. ff-HEDM cannot provide spatial information to the DSGODs, but a CIP signature, once validated through the electron characterization methods listed above, can be tracked in-situ for large collections of both surface and subsurface grains as they evolve under cyclic loading. The data in [8], along with these additional characterizations, provide a one-of-a-kind opportunity to explore our hypothesis.

In this study, we utilize this strategic combination of electron and X-ray characterization techniques to make in-situ measurements of bulk Inconel-718 material to objectively identify and track grains prior to crack initiation, under the hypothesis that DSGODs can act as a signature of grains with high CIP. While a fatigue crack was not confirmed in these data, our study is based on a well-studied crack initiation theory in nickel-based superalloys and focuses on the grain-scale cyclic plasticity that serves as the precursor to fatigue failure $[6,7]$. Our approach consists of four sequential steps:

1. Discovering a signature of CIP: we construct DSGODs for each grain in a surface measurement to discover and validate DSGOD statistics as a CIP signature through the ground-truth data of 2DEBSD and H-DIC measurements,

2. Verifying a CIP signature in material's subsurface: we construct DSGODs and their statistics for each grain in a 3D microstructure characterized by TriBeam to verify a CIP signature in a material's subsurface,

3. Confirming CIP signature detection through forward projection simulation: we model the 3D microstructure measured by TriBeam in a virtual diffraction simulation $[19,24,25]$ to assess the feasibility of observing a CIP signature in ff-HEDM measurements,

4. Tracking the evolution of a CIP signature in-situ: we conduct an in-situ cyclic ff-HEDM experiment to characterize the evolution of grains with a CIP signature under low-cycle fatigue conditions.

Utilizing this multimodal approach, we methodically link each characterization technique in search of a signature to focus fatigue studies to further the understanding of when and where grain-scale cyclic plasticity leads to the initiation of a fatigue crack. Signatures, like the CIP signature discovered here, will open new strategies for studying fatigue crack initiation.

In this paper, italicized letters (a) indicate scalars, bold lower-case letters (a) indicate vectors, and bold upper-case letters $(\mathbf{A})$ indicate tensors of rank two and above. For any tensor, the magnitude of the tensor is expressed as

$$
\|\mathbf{A}\|=(\mathbf{A}: \mathbf{A})^{\frac{1}{2}} .
$$


We will use the words "crystal" and "grains" interchangeably throughout the paper. We describe lattice orientations as the transformation from a crystal coordinate system to the sample coordinate system. We use the Rodrigues parameterization of orientation space to represent orientations [26]. A Rodrigues orientation vector $(\mathbf{r})$ is defined as

$$
\mathbf{r}=\mathbf{n} \tan \left(\frac{\phi}{2}\right)
$$

where the unit vector parallel to the axis of rotation is $\mathbf{n}$ and the rotation angle about the axis of rotation is $\phi$. A point in Rodrigues orientation space is equivalent to a crystallographic orientation.

\section{Results}

\section{Discovering a signature of CIP.}

To begin our investigation, we collected in-situ surface H-DIC and 2D-EBSD measurements on a polished Inconel-718 sample that has been loaded in tension to a macroscopic strain of $1.26 \%$. Figure 3(a) presents the surface microstructure of the sample. With the orientation information provided through 2D-EBSD, the EBSD DSGOD reconstruction method (See Methods) computed the DSGODs of 683 grains. These grains are referenced as $2 D$-EBSD grains in this study. We calculated the higher order statistical moments of each DSGOD (See Methods), resulting in two scalar metrics for each DSGOD: the skewness magnitude $(\|\boldsymbol{\Gamma}\|)$ related to the asymmetry of a DSGOD and the kurtosis magnitude $(\|\mathbf{K}\|)$ related to the relative weight of the tails of a DSGOD [14]. We elected to use $\|\boldsymbol{\Gamma}\|$ and $\|\mathbf{K}\|$ to serve as the CIP signature to identify grains with microvolumes given the skewed and long-tailed morphology of a DSGOD of a grain with microvolume presence, like Grain A presented in Figure 2. The histogram and correlation plots of $\|\boldsymbol{\Gamma}\|$ and $\|\mathbf{K}\|$ for the 2D-EBSD grains are presented in Figure 3(b). The histograms depict large, asymmetric peaks with long tails toward the larger values of $\|\boldsymbol{\Gamma}\|$ and $\|\mathbf{K}\|$ while the correlation plot details individual grains with larger values of $\|\boldsymbol{\Gamma}\|$ and $\|\mathbf{K}\|$ separated from the main grouping. The characteristics of these plots seem to indicate differences in intragrain lattice rotation between the majority of grains and the small collection of grains deviating from the majority. To quantify the grouping behavior of grains with differing DSGOD statistics, we applied the K-means clustering algorithm to the values of $\|\boldsymbol{\Gamma}\|$ and $\|\mathbf{K}\|$ across all the grains in the 2D-EBSD analysis with the results presented in Figure 3(c) [27]. The clustering algorithm identified a larger cluster of low skewness - low kurtosis (LS-LK) grains and a smaller cluster of high skewness - high kurtosis (HS-HK) grains with each cluster containing 636 grains and 47 grains respectively.

We investigate the difference in DSGOD statistics further by offering an in-depth study of one HS-HK grain of interest in the 2D-EBSD HS-HK cluster. Figure 3 presents the DSGOD of Grain E (d) as well as the 
misorientation (e) and strain (f) fields near Grain E. The DSGOD of Grain E shares a skewed, long-tailed distribution similar to Grain A that had a microvolume in Figure 2(a-b). The CIP signature in Grain E is confirmed with the ground-truth misorientation field which depicts a microvolume within Grain E. The strain field in the same region of intersection shows a slip localization event that terminates at the neighboring grain boundary, reaffirming the CIP signature and the propensity for crack initiation.

Although this in-depth study included only one HS-HK grain, manual inspection of the 2D-EBSD and H-DIC measurements definitively identified 16 other grains containing microvolumes with 3 microvolume grains classified as LS-LK and 13 microvolume grains classified as HS-HK grains (See Supplementary Note 1 and 2). These results from the 2D-EBSD grains demonstrate the capabilities of DSGOD statistics serving as a CIP signature to identify grains with microvolumes, theorized to be grains likely to initiate fatigue cracks. 

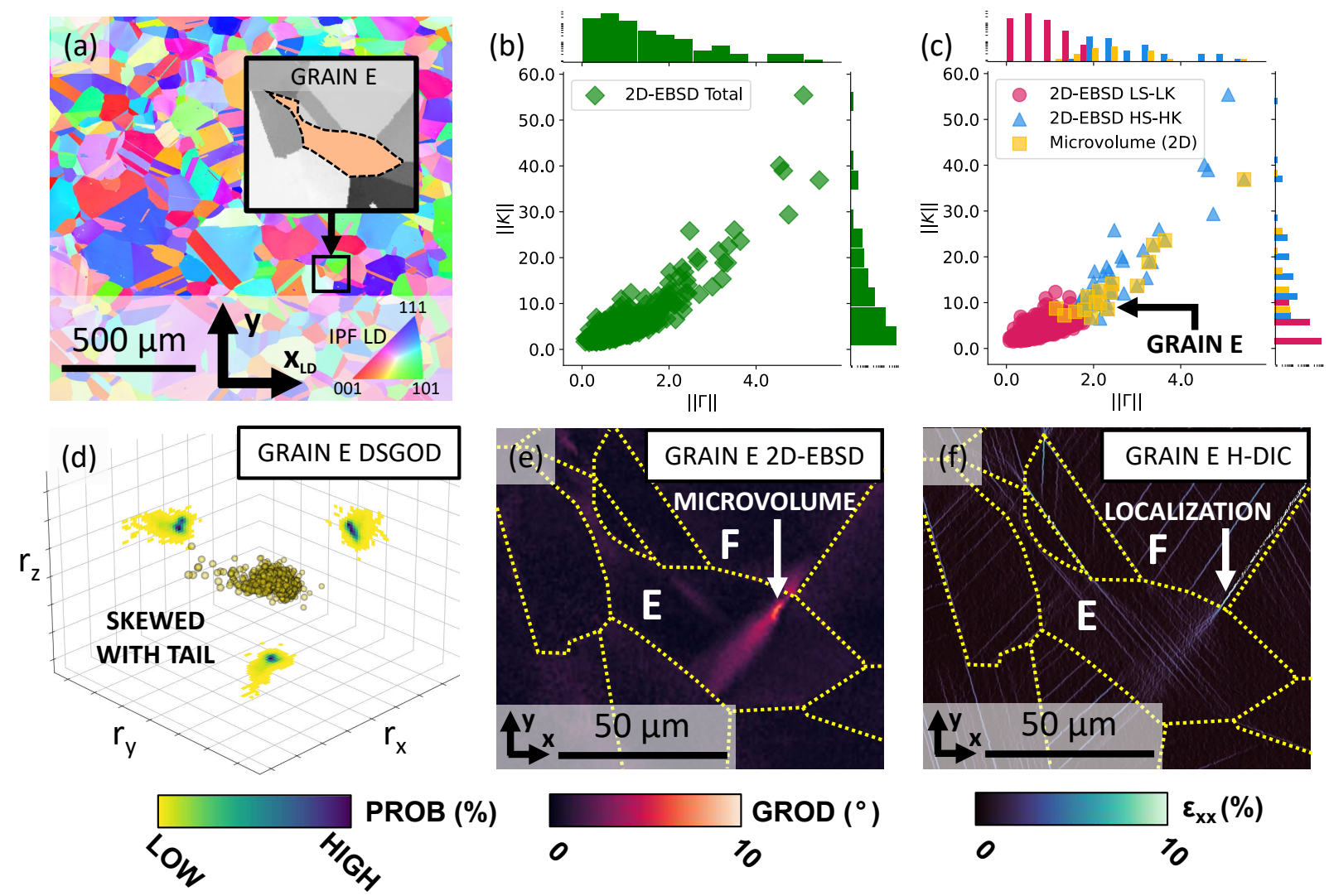

Figure 3: (a) Inverse pole figure map aligned with the macroscopic loading direction $\left(\mathrm{x}_{\mathrm{LD}}\right)$ displaying orientation information from 2D-EBSD measurements on the surface of the Inconel-718 material deformed in tension to a macroscopic strain of $1.26 \%$ (same sample as Figure 1 and Figure 2). Inset showing Grain E, found via DSGOD statistics, outlined by a dashed black line in its grain neighborhood. (b) The DSGOD statistics $(\|\mathbf{\Gamma}\|$ and $\|\mathbf{K}\|)$ for each grain in the 2D-EBSD dataset presented as scatter and logarithmic histograms. (c) 2D-EBSD DSGOD statistics clustered via K-means to identify the low skewness - low kurtosis (LS-LK) and high skewness - high kurtosis (HS-HK) grain clusters. The LS-LK cluster contains 636 grains and the HS-HK cluster contains 47 grains. The DSGOD statistics corresponding to Grain E are marked in the plot as an example of a HS-HK grain. (d) DSGOD of Grain E presented in a reduced region of Rodrigues orientation space with 2D projected densities on the far faces of each plot area. (e) 2D-EBSD misorientation field colored by GROD depicting Grain E and neighboring grains, with grain boundaries outlined in yellow. (f) H-DIC strain field of the same neighborhood of Grain E, with grain boundaries outlined in yellow. The clustering indicated Grain E shared the CIP signature through higher values of DSGOD statistics. The ground-truth data in the GROD field (e) and the strain field (f) confirms the CIP signature with the observation of a microvolume in Grain E caused by the slip localization event in Grain F and indicates a favorable site for crack initiation.

\section{Verifying a CIP signature in material's subsurface.}

The previous section discovered that DSGOD statistics can be utilized as signature of grains with high CIP on the surface of a material. We then validated this signature by using DSGOD statistics to identify grains of interest and manually confirming the presence of a microvolume and slip localization via the ground-truth 
orientation and microscopic strain fields. The next step in our approach works to verify the effectiveness of using this signature to identify subsurface grains with high CIP.

Three-dimensional orientation data of the same Inconel-718 sample were acquired using Tribeam tomography [15] in a region where 2D-EBSD and H-DIC surface measurements were performed (See Methods) [28]. The TriBeam microstructure is presented in Figure 4(a). The EBSD DSGOD reconstruction method computed the DSGODs of 1,667 grains. These grains are referenced as TriBeam grains for this study. The histogram and correlation plots of $\|\mathbf{\Gamma}\|$ and $\|\mathbf{K}\|$ for the TriBeam grains are presented in Figure 4(b). These results demonstrate that subsurface grains measured via TriBeam share similar trends in DSGOD statistics as the surface grains measured via 2D-EBSD by possessing a large collection LS-LK grains and a small collection of HS-HK grains. K-means clustering quantified these collections by clustering values of $\|\boldsymbol{\Gamma}\|$ and $\|\mathbf{K}\|$ presented in Figure 4(c). The clustering algorithm identified 1487 grains in the LS-LK cluster and 180 grains in the HS-HK cluster.

To verify the CIP signature in HS-HK subsurface grains, we selected Grain G from the TriBeam HS-HK cluster to investigate in further detail. Figure 4(d, e) presents the DSGOD and the spatial mapping of the misorientation field of Grain G. The DSGOD of Grain G has higher values of $\|\boldsymbol{\Gamma}\|$ and $\|\mathbf{K}\|$, indicative of the CIP signature, and shares a skewed and long-tailed distribution similar to grains with microvolumes such as Grain A in Figure 2(a-b) and Grain E in Figure 3(d-f). The ground-truth misorientation field of Grain $G$ verifies the presence of a microvolume in Grain G depicted by a streak of concentrated lattice rotation lying near the grain boundary. The microscopic strain fields cannot be reconstructed in the subsurface with these methods to confirm the neighboring slip localization event, yet the misorientation behavior of Grain E suggests that HS-HK subsurface grains experience the same neighboring localized slip as seen on the surface through H-DIC and 2D-EBSD measurements presented prior. Based on this inference, we identified 29 subsurface grains sharing similar regions of concentrated intragrain lattice rotation presumed to be microvolumes in the TriBeam measurement. Of these presumed microvolume grains, 9 grains were classified as LS-LK and 20 grains were classified as HS-HK (See Supplementary Note 1 and 3). Given these results, DSGOD statistics, calculated from DSGODs of subsurface grains in the TriBeam measurement, are signatures of grains with high CIP, precisely like the DSGOD statistics from the 2D-EBSD surface measurements. 


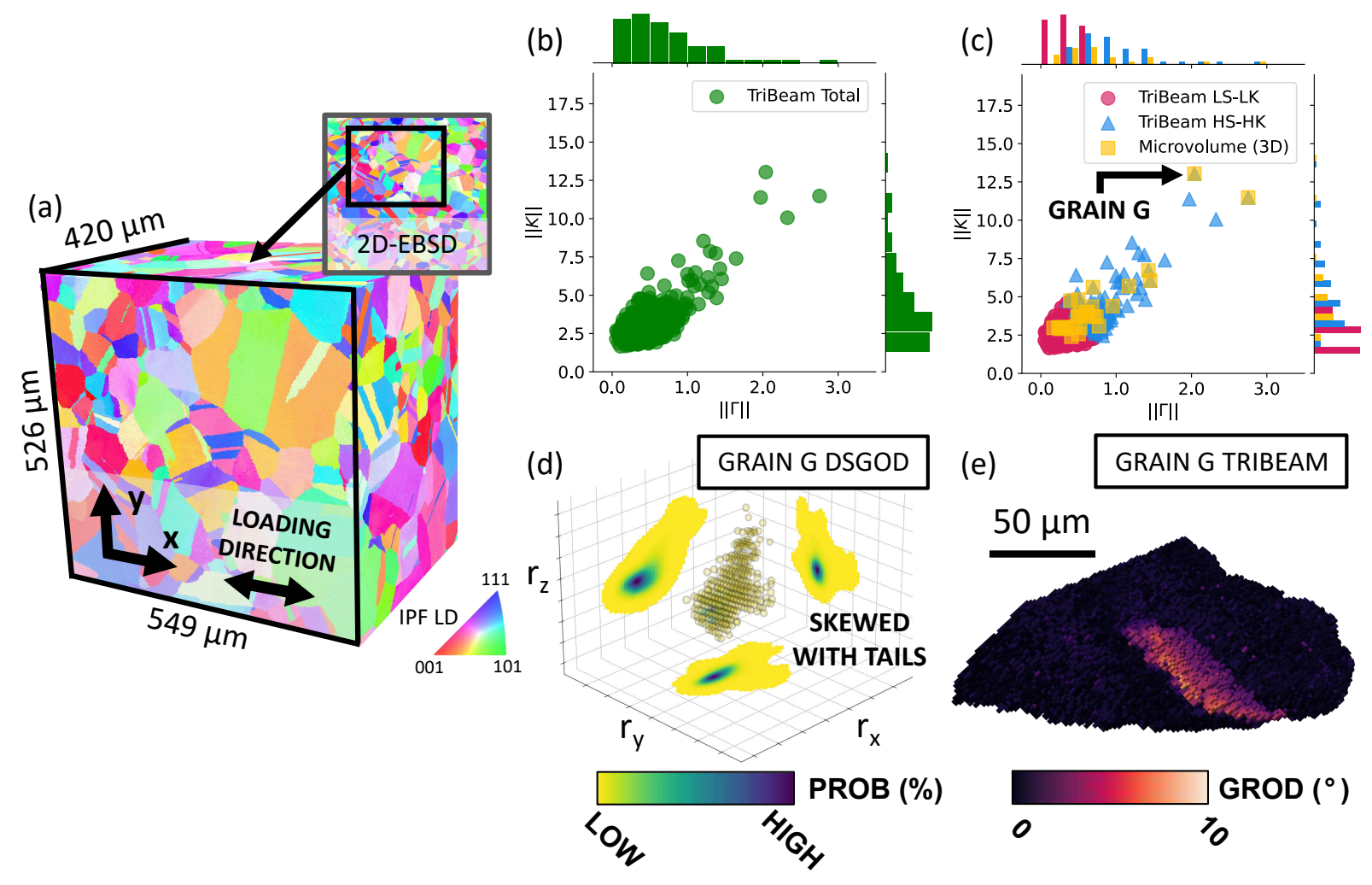

Figure 4: (a) Inverse pole figure map aligned with the macroscopic loading direction displaying orientation information from volumetric TriBeam measurement of the Inconel-718 material deformed in tension to a macroscopic strain of $1.26 \%$ (same sample as Figure 1, Figure 2, and Figure 3). Inset showing portion of the 2D-EBSD measurement where the TriBeam measurement was collected. (b) DSGOD statistics $(\|\boldsymbol{\Gamma}\|$ and $\|\mathbf{K}\|)$ for each grain in the TriBeam dataset presented as scatter and logarithmic histograms. (c) TriBeam DSGOD statistics clustered via K-means to identify the low skewness - low kurtosis (LS-LK) and high skewness - high kurtosis (HS-HK) grain clusters. The LS-LK cluster contains 1487 grains and the HS-HK cluster contains 180 grains. The DSGOD statistics corresponding to subsurface Grain G are marked in the plot as an example of a HS-HK grain. (d) DSGOD of Grain G presented in a reduced region of Rodrigues orientation space with 2D projected densities on the far faces of each plot area. (e) 3D spatial morphology of Grain G colored by GROD. The DSGOD statistics and clustering indicated Grain G had the CIP signature. The ground-truth data in the GROD field (e) confirmed the CIP signature with the observation of a microvolume in Grain G.

\section{Confirming CIP signature detection through forward projection simulation.}

The previous two steps in this approach discovered and verified DSGOD statistics serving as signatures of CIP both on the surface and in the subsurface of a material. However, these EBSD methods were either limited to a material surface or limited to a static and forensic investigation of the material without any means of characterizing material evolution under loading conditions like cyclic deformation. The ff-HEDM technique provides the in-situ measurements of the response of bulk material necessary to understand the interaction between grain-scale cyclic plasticity and crack initiation through the CIP signature. 
Before assuming the CIP signature can be resolved in DSOGDs reconstructed from ff-HEDM measurements, we seek to first validate the current HEDM DSGOD reconstruction method (See Methods) and DSGOD statistics by modeling the ground-truth spatial and orientation information provided by TriBeam measurements in a simulated X-ray diffraction experiment via a virtual diffractometer [19,24,25]. A virtual diffractometer (VD) employs a forward projection model to simulate a diffraction experiment by projecting X-rays through a virtual sample and onto a virtual detector by accounting for crystal structure, instrument setup, and experimental geometry. With this strategy, the DSGOD of each grain in the TriBeam microstructure can be reconstructed with the ff-HEDM DSGOD reconstruction method using the synthetic diffraction data from the virtual diffraction simulation. These ff-HEDM DSGODs can then be directly compared to the ground-truth DSGODs of the exact same grains in the TriBeam measurement reconstructed with the EBSD DSGOD reconstruction method. Furthermore, the spatially-resolved orientation fields of the TriBeam measurement can serve as a visual confirmation of grains whose ff-HEDM DSGOD statistics indicate microvolume presence, and therefore high CIP.

To start this analysis, a virtual microstructure created from the TriBeam measurement was modeled in a synthetic ff-HEDM experiment via a VD (See Methods). The ff-HEDM DSGOD reconstruction method applied to the synthetic diffraction data computed the DSGODs of 1,254 grains ( $75 \%$ of the original TriBeam grains) and are referenced as VD TriBeam grains for this study. The histogram and correlation plots of $\|\boldsymbol{\Gamma}\|$ and $\|\mathbf{K}\|$ for the VD TriBeam data are presented in Figure 5(a). Trends of LS-LK grains and HS-HK grains in the VD TriBeam DSGOD statistics show similarities to the 2D-EBSD and the TriBeam trends. K-means clustering of $\|\boldsymbol{\Gamma}\|$ and $\|\mathbf{K}\|$ for the VD TriBeam grains quantified the LS-LK and HS-HK clusters with 1025 grains and 229 grains respectively, as shown in Figure 5(b). We note that K-means clustering is an unsupervised algorithm and involves no knowledge between the TriBeam and VD TriBeam datasets when clustering. Despite this information, both the TriBeam and the VD TriBeam clusters were of similar proportions of $\|\mathbf{\Gamma}\|$ and $\|\mathbf{K}\|$. All 30 subsurface grains with presumed microvolumes from the TriBeam grains were able to be reconstructed in the simulation and are plotted with their VD TriBeam DSGOD statistics. Of these presumed microvolume grains, 14 grains were classified as LS-LK and 16 grains were classified as HS-HK (See Supplementary Note 1 and 3).

To explore the differences in results of the EBSD and ff-HEDM DSGOD reconstruction methods applied to these data, the same grain from the TriBeam results (Grain G) was selected for further analysis. When comparing the statistics of Grain G in the clustering plots of Figure 4(c) and Figure 5(b), the ff-HEDM DSGOD reconstruction method produces a DSGOD with smaller values of $\|\boldsymbol{\Gamma}\|$ and $\|\mathbf{K}\|$. Figure 5 (c-d) visually demonstrates this difference in results of the DSGOD reconstruction methods of Grain G. The EBSD DSGOD reconstruction method has accurately resolved the orientations on the extremities of the TriBeam 
DSGOD while the ff-HEDM DSGOD reconstruction method does not, resulting in a smaller, less defined VD TriBeam DSGOD. The difference in reconstruction quality can be readily explained by the abilities of each experimental technique. The TriBeam system individually probes small regions $\left(1 \mu m^{3}\right)$ within a material through serial sectioning, while the ff-HEDM technique often probes larger regions $\left(10.1-1 \mathrm{~mm}^{3}\right)$ of a material at one time, without the luxury of high resolution spatial information to measure the extremities of a DSGOD. Despite the difference in experimental capabilities, the ff-HEDM DSGOD reconstruction method still offers an excellent approximation of the ground-truth single grain orientation distribution as shown through direct comparisons of DSGODs and similar clustering behavior in both techniques. In the interest of identifying the CIP signature, the ff-HEDM DSGOD reconstruction method is a highly suitable technique for identifying grains with high CIP. 
(a)
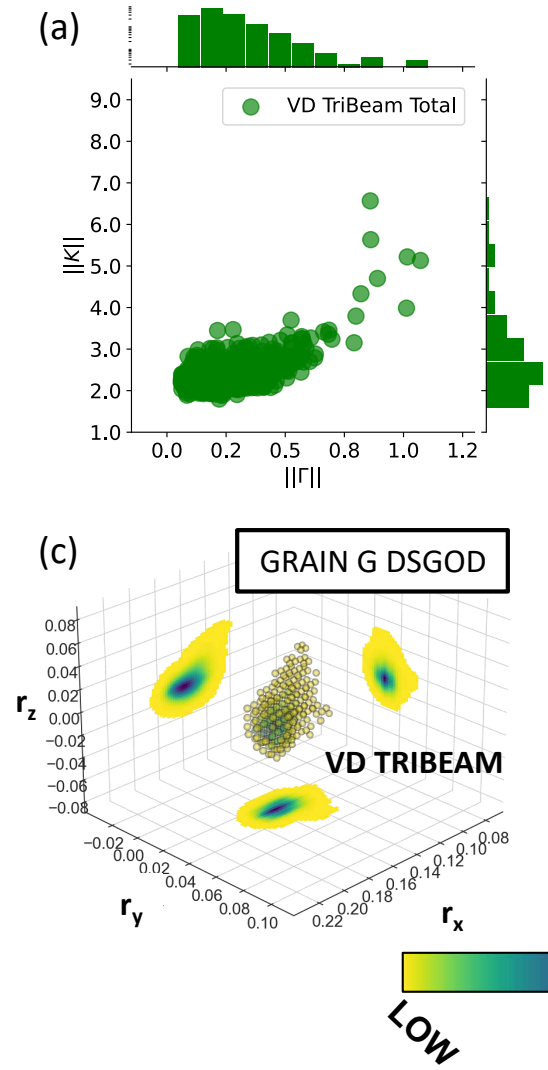

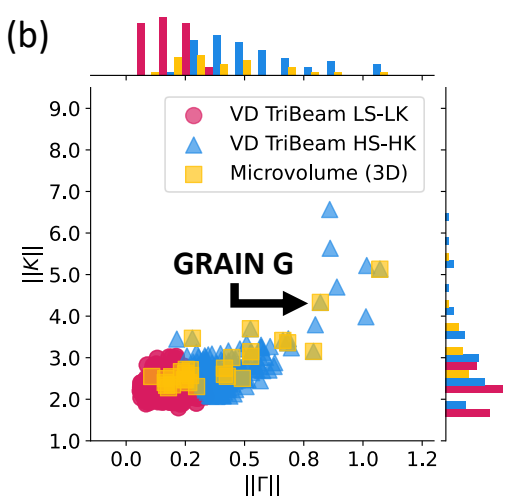

(d)

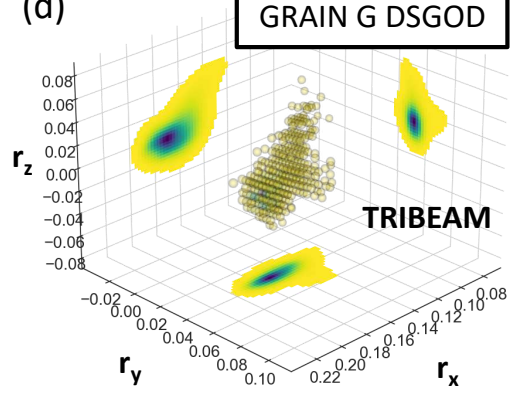

PROB (\%)

Figure 5: (a) DSGOD statistics $(\|\boldsymbol{\Gamma}\|$ and $\|\mathbf{K}\|)$ for each grain in the VD TriBeam dataset presented as scatter and logarithmic histograms. (b) Same VD TriBeam DSGOD statistics clustered via K-means to identify the low skewness - low kurtosis (LS-LK) and high skewness - high kurtosis (HS-HK) grain clusters. The LS-LK cluster contains 1025 grains and the HS-HK cluster contains 229 grains. The statistics corresponding to the same subsurface Grain G from Figure 4 are marked as an example of a HS-HK grain. We note the different axes scales in plots when compared to Figure 4(b-c). (c) DSGOD of Grain G from the TriBeam measurement reconstructed from the VD TriBeam data using the ff-HEDM DSGOD reconstruction method with 2D projected orientation densities on the far faces of each plot area. (d) DSGOD of the same Grain G reconstructed from the TriBeam data using the EBSD DSGOD reconstruction method with 2D projected orientation densities on the far faces of each plot area.

\section{Tracking the evolution of a CIP signature in-situ.}

With the previous three steps discovering, verifying, and confirming DSGOD statistics as a signature of CIP in grains above and below the surface of a material, we conducted an in-situ cyclic ff-HEDM experiment at the FAST beamline at the Cornell High Energy Synchrotron Source (See Methods). A schematic of the sample and experiment are provided in Figure 6(a). In the experiment, a total of 1,002 grains were indexed and fit in the initial, undeformed diffraction volume of a Inconel-718 sample. We note that this sample is different from the previous sample characterized via 2D-EBSD and TriBeam. We collected in-situ ff-HEDM measurements of the same diffraction volume at 14 pre-determined load steps along stress-strain curve of two 
fully-reversed loading cycles, shown in Figure 6(b). The ff-HEDM DSGOD reconstruction method extracted the DSGODs of 507 grains at every measurement in the experiment, which are referenced as HEDM grains for this study. We chose to limit further analysis to these 507 HEDM grains due to insight gained by monitoring how grains and their DSGODs evolve at each load step around a hysteresis loop and from one cycle to the next. In addition to the DSGOD reconstructions, the diffraction events of each HEDM grain were fit to calculate grain-averaged position, orientation, and elastic strain tensor at each measurement.

Figure 6(c-d) presents $\|\mathbf{\Gamma}\|$ and $\|\mathbf{K}\|$ histogram and correlation plots for the HEDM grains over a few select load steps in the deformation history where ff-HEDM measurements were collected. At each load step, K-means clustering quantified the clustering behavior $\|\mathbf{\Gamma}\|$ and $\|\mathbf{K}\|$ for each grain. Figure 6(e-f) presents the clustering results for the same select load steps where ff-HEDM measurements were collected with the distributions of $\|\boldsymbol{\Gamma}\|$ and $\|\mathbf{K}\|$ resembling the statistical trends from the previous 2D-EBSD and TriBeam analyses. Although the results of all the ff-HEDM measurements are not depicted here, manual inspection showed that most distributions shared similar trends in grouping behavior with a large collection of LS-LK grains and a small collection of grains HS-HK (See Supplementary Note 4). This behavior was consistent despite all the measurements capturing different instances in the loading history. We note that grains did not always remain in their initial clustering of LS-LK grains or HS-HK grains. Grains could move between clusters as deformation progressed and their DSGOD statistics evolved. 

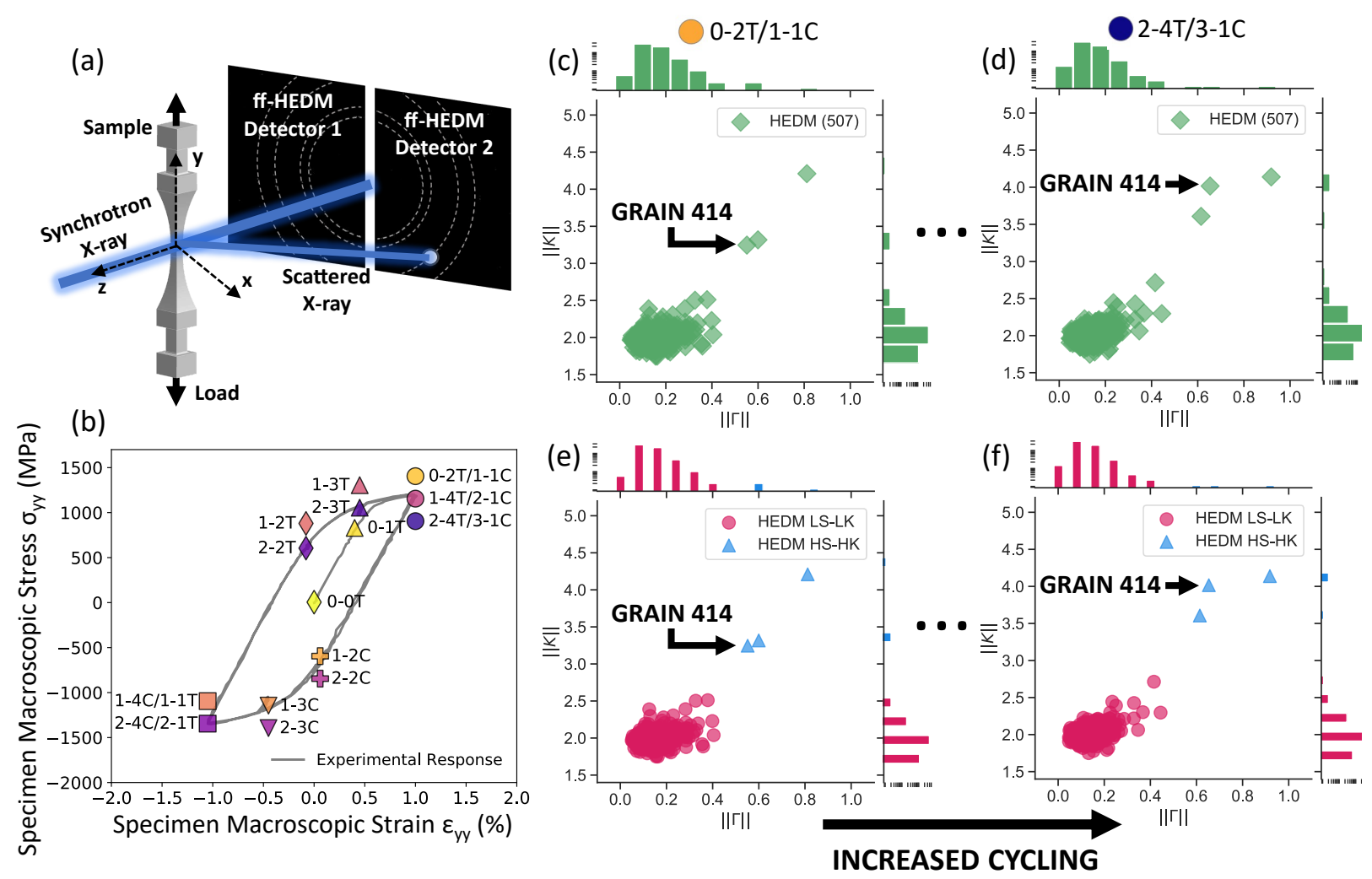

Figure 6: (a) A schematic of the ff-HEDM Inconel-718 sample and the ff-HEDM experiment. The sample was cyclically loaded in the $y$-direction as indicated by the arrows above and below the sample. (b) The macroscopic stress-strain curve of the sample from two loading cycles. The macroscopic stress in the loading direction is given by $\sigma_{y y}$ and the macroscopic strain in the loading direction is given by $\epsilon_{y y}$. The individual load steps where ff-HEDM measurements were collected are marked by a colored shape and label. The label for a given measurement is $\mathrm{X}-\mathrm{YZ}$ where $\mathrm{X}$ is the cycle number, $\mathrm{Y}$ is the cycle sub-step, and $\mathrm{Z}$ indicates the direction of loading where the measurement was taken with tension as " $\mathrm{T}$ " or compression as " $\mathrm{C}$ ". Any label with a "/" indicates one measurement classified under transitioning parts of the cycle. (c-d) A sampling of the DSGOD statistics $(\|\boldsymbol{\Gamma}\|$ and $\|\mathbf{K}\|)$ for each grain available at each loadstep in the HEDM dataset presented as scatter and logarithmic histograms. (e-f) Same sampling of DSGOD statistics clustered via K-means to identify low skewness - low kurtosis (LS-LK) and high skewness - high kurtosis (HS-HK) grain clusters. For $0-2 \mathrm{~T} / 1-1 \mathrm{C}$ and $2-4 \mathrm{~T} / 3-1 \mathrm{C}$, the LS-LK clusters contain 504 grains and the HS-HK clusters contain 3 grains.

In contrast to the prior EBSD measurements, ff-HEDM measurements provide grain-averaged stress information that can be used to investigate the evolution of deformation that could drive the CIP signature [29]. In an effort to preserve the time-resolved information of the HEDM grains, we offer a case study of one HS-HK grain (Grain 414) present in four HS-HK clusters throughout the loading history that exhibits the CIP signature. Orientation and spatial neighborhood dictate the mechanical response of a grain and its neighbors, as indicated in Figure 1. Therefore, we include grains that are neighboring Grain 414 as a comparison. Since exact grain topology cannot be mapped via ff-HEDM, we define neighbors as grains whose center-of-mass positions are within $100 \mu \mathrm{m}$ of the Grain 414 [21]. A total of 14 neighboring HEDM 
grains met this criteria. Figure 7 presents an assortment of metrics for Grain 414 and its neighbors against each load step where ff-HEDM measurements were collected. These metrics include $\|\boldsymbol{\Gamma}\|$ and $\|\mathbf{K}\|$ as well as stress metrics obtained through the elastic strain tensors calculated from fitting the ff-HEDM data [21]. Table 1 (See Methods) provides the definitions for each of the stress metrics used in Figure 7. The metrics were chosen to provide meaningful information related to the driving forces behind DSGOD evolution, CIP signature, and potentially crack initiation.
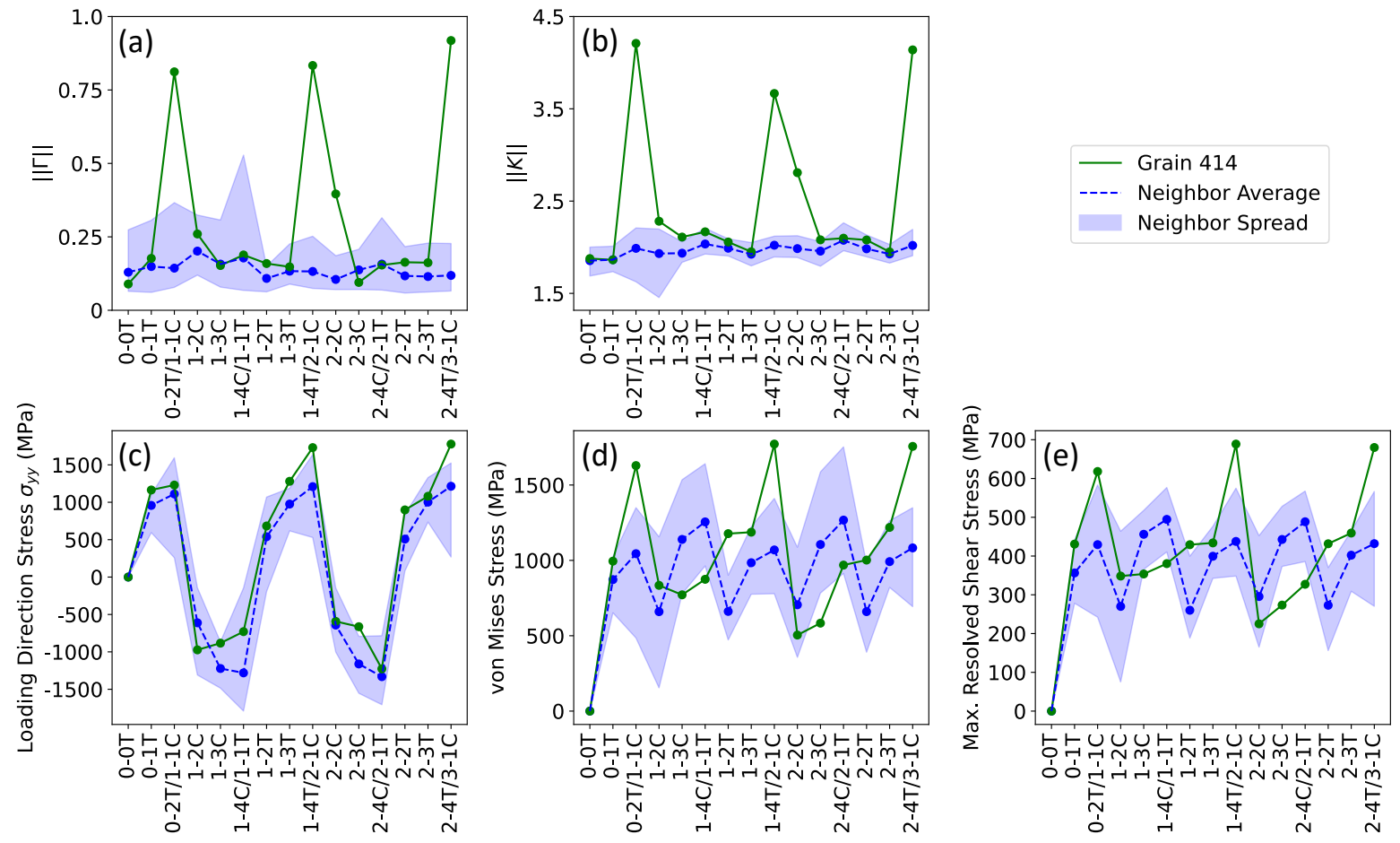

Figure 7: DSGOD statistics $(\|\boldsymbol{\Gamma}\|$ and $\|\mathbf{K}\|)$ and stress metrics (See Methods) for Grain 414 and neighboring grains plotted against the load steps where ff-HEDM measurements were collected. The trends of Grain 414 are represented as a solid green line. The trends of neighboring grains are represented by an average plotted as a dotted blue line and as a spread represented as the area between the maximum and minimum value of the neighbors for each load step shaded with light blue. In this context, neighbors are defined as any grain whose center-of-mass position is within $100 \mu \mathrm{m}$ of the center-of-mass position of Grain 414. Grain 414 was clustered as a high skewness - high kurtosis (HS-HK) grain at load steps 0-2T/1-1C, 1-4T/2-1C, 2-2C, and $2-4 \mathrm{~T} / 3-1 \mathrm{C}$ on the macroscopic stress-strain curve due to the skewed, long-tailed DSGODs present at these load steps as seen in Figure 8.

Upon examining Figure 7(a-b), Grain 414 experienced different intragrain lattice rotation than its neighboring grains, especially in the maximum macroscopic tensile load steps or tension tips of cycling. The $\|\boldsymbol{\Gamma}\|$ and $\|\mathbf{K}\|$ values of Grain 414 sharply increased at each of the tension tips before returning to a state near the neighbor average upon load reversal into compression. Interestingly, the maximum macroscopic compressive load steps or compression tips of cycling did not share this same behavior. To reason the difference 
between the tension and compression tips, we look to the stress metrics. Figure $7(\mathrm{c})$ presents the plot of the stress component along the macroscopic loading direction of each grain, $\sigma_{y y}$, for Grain 414 and its neighbors (grains within $100 \mu \mathrm{m}$ of the Grain 414). When compared to its average neighbor, Grain 414 exhibited larger magnitudes of $\sigma_{y y}$ at each of the tension tips and smaller magnitudes of $\sigma_{y y}$ at one of the compression tips. Figure $7(\mathrm{~d})$ presents the plot of von Mises stress, a measure of nearness to the macroscopic yield surface, and magnifies the differing cyclic behavior of Grain 414 and its neighbors. Grain 414 displayed higher amounts of von Mises stress at the tension tips whereas the neighboring grains displayed higher amounts of von Mises stress during the compression tips. Figure $7(\mathrm{e})$ presents the maximum resolved shear stress magnitude of each grain, which is a measure of grain-scale yielding by projecting the grain-averaged stress onto the slip systems of the grain. The trend for maximum resolved shear stress magnitude followed a similar pattern to the von Mises stress in which Grain 414 displayed largest magnitudes at the tension tips and the neighboring grains displayed largest magnitudes at the compression tips. The opposing, cyclic behavior between Grain 414 and the neighboring grains expresses the heterogeneous spread of deformation in this local neighborhood. Of particular interest are the larger magnitudes of stresses in Grain 414 in the tension tips when the crack formation process is most active, indicating favorable conditions for fatigue crack initiation and reaffirming the CIP signature.

To better visualize the evolution of Grain 414, Figure 8 depicts the DSGOD of Grain 414 at different load steps around the loading cycle (See Supplementary Movie 1). We note the long-tailed, skewed distributions observed in the tension tips directly correspond to the high values of $\|\mathbf{\Gamma}\|$ and $\|\mathbf{K}\|$ calculated at the same load steps and are similar to the DSGODs of grains with microvolumes shown in Figure 2(a-b), Figure 3(d-f), and Figure 4(d-e), indicative of the CIP signature. 


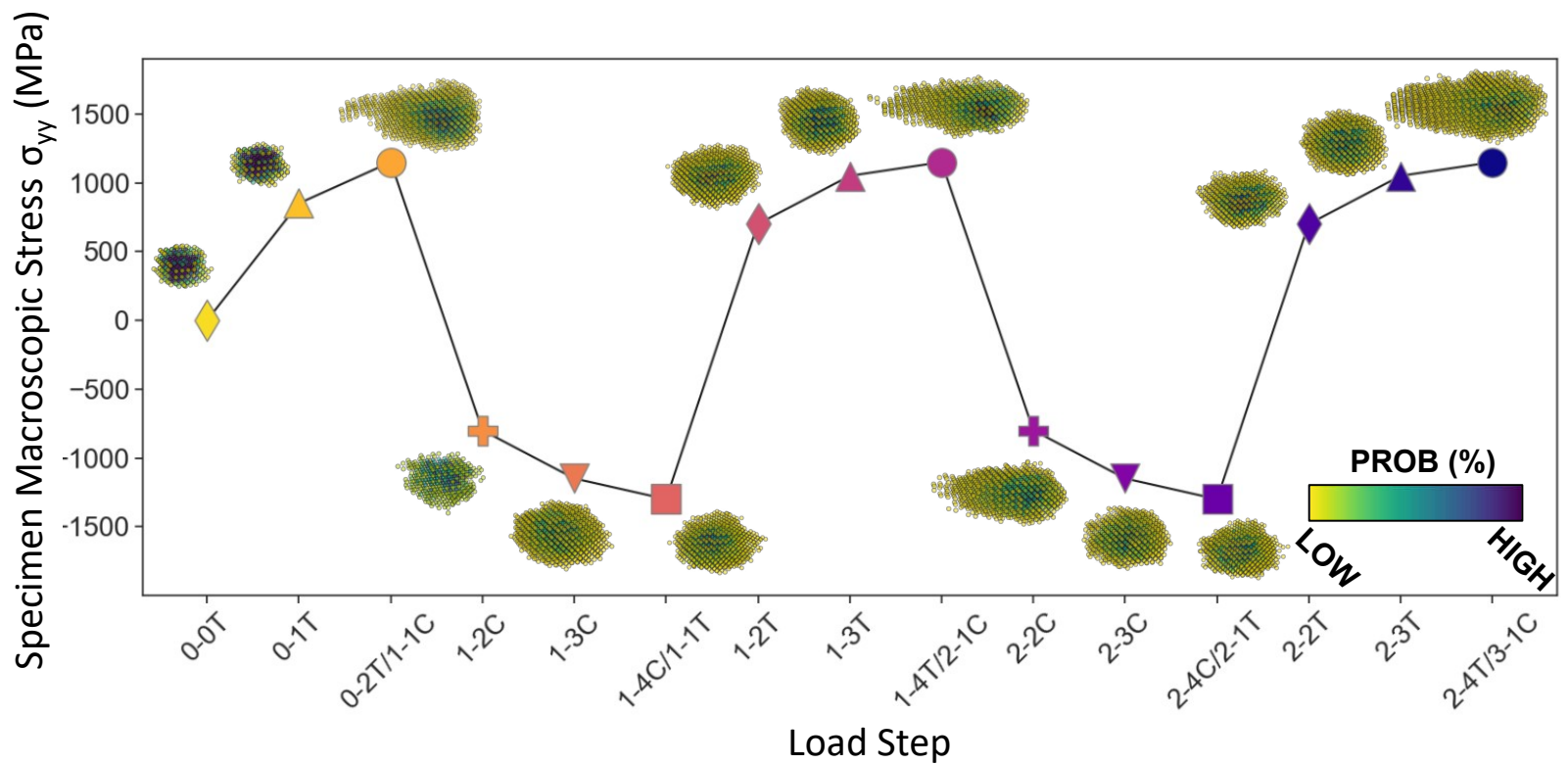

Figure 8: The macroscopic stress in the loading direction $\sigma_{y y}$ as a function of load step where ff-HEDM measurements were collected. The colored shapes correspond to the load steps in the macroscopic stressstrain curve presented in Figure 6. The evolution of DSGODs for Grain 414 from the ff-HEDM dataset is plotted in a reduced region of Rodrigues orientation space for each ff-HEDM measurement. Grain 414 was clustered as a high skewness - high kurtosis (HS-HK) grain at the tension tips of the macroscopic stress-strain curve due to the skewed, long-tailed DSGODs present at these load steps.

The combination of the DSGOD statistics and stress metrics throughout the loading history provide unique information on the state of deformation and loading of a single grain. These methods are not limited to one grain, but are accessible to every grain in the aggregate that can be captured in the diffraction signal. With these DSGOD statistics serving as a signature, we have focused our in-situ study of fatigue crack initiation from over 1000 grains to one high CIP grain.

\section{Discussion}

In this study, we discovered a CIP signature based on two key connections. First, the crack initiation theory of Villechaise et al and Larrouy et al connected crack initiationb in nickel-based superalloys to the impingement of slip localization on a grain boundary where slip transmission is not facilitated [6,7]. Second, Stinville et al used the microscopic strain and orientation fields provided by H-DIC and 2D-EBSD surface measurements to directly connect the impingement of slip localization physically shearing a neighboring grain boundary to microvolumes [8]. With this information at hand, we conducted a multimodal approach that combined the microscopic strain fields of surface H-DIC measurements and orientation fields of surface and volumetric EBSD measurements with the time-resolved orientation and grain-scale mechanical information 
of ff-HEDM measurements to investigate the effectiveness of using the statistics of DSGODs as a signature of grains with with high CIP. In the 2D-EBSD measurement, we identified a small collection of grains by computing the DSGODs and their statistics $(\|\mathbf{\Gamma}\|,\|\mathbf{K}\|)$ from the orientation information available for each grain. This small collection of grains exhibited higher values of $\|\boldsymbol{\Gamma}\|$ and $\|\mathbf{K}\|$ than the average grain in the sample. Inspection of these HS-HK grains through 2D-EBSD and H-DIC determined that the majority of grains with microvolumes in the measurement were HS-HK grains. These statistics defined our CIP signature as the connection between microvolume presence and higher values of $\|\boldsymbol{\Gamma}\|$ and $\|\mathbf{K}\|$. The TriBeam measurements confirmed the CIP signature existed in the subsurface of the material. The ground-truth TriBeam orientation information was modeled in a synthetic ff-HEDM experiment via a virtual diffractometer to validate the capabilities of DSGOD reconstruction in ff-HEDM measurements, which have the advantage of collecting orientation and mechanical information in real-time via in-situ measurements of bulk material. With the knowledge of the previous steps in the approach, our study culminated in an in-situ ff-HEDM experiment that tracked 507 grains and their DSGODs in an Inconel-718 sample through two fully-reversed loading cycles. The in-situ measurements captured the evolution of DSGOD statistics around a hysteresis loop and from cycle to cycle to identify a HS-HK grain. The grain-averaged mechanical information available through ff-HEDM demonstrated this HS-HK grain exhibited a vastly different stress state than neighboring grains, specifically at the maximum tensile load where crack formation is most active. These results bring confidence to the CIP signature's effectiveness of focusing the study of fatigue crack initiation.

Even though the foundations of fatigue failure still remain challenging, this study pursues a next step in the direction of unraveling this daunting problem. The newly discovered CIP signature serves as a data search criterion tool for identifying "at-risk" grains with the greater inferred potential to initiate a fatigue crack. An exciting opportunity arises from this CIP signature when used in conjunction with real-time, insitu ff-HEDM measurements. During a cyclic loading experiment, one can actually "watch" a grain develop its CIP signature and monitor the most at-risk grain as it evolves throughout loading cycles. As seen in the HEDM results presented here, a grain with the CIP signature can develop higher values of $\|\mathbf{\Gamma}\|$ and $\|\mathbf{K}\|$ of the CIP signature at some load steps and reverse behavior to lower values of $\|\boldsymbol{\Gamma}\|$ and $\|\mathbf{K}\|$ at other load steps (See Figure 7). However, these results only begin to tap into the richness of an HEDM dataset to understand challenging processes like fatigue crack initiation. Grain-averaged stresses and strains provide the initial information to describe favorable fatigue crack conditions, but perhaps the enormous promise is going beyond averages and extracting intragrain distributions of stress and strain for each grain from the diffraction data $[23,30]$. Using single grain orientation, stress, and strain distributions derived from experimental data and linking to sophisticated crystal plasticity modeling could supply the information to more fully understand the process of fatigue crack initiation. 
The general form of this signature discovery process is not limited to the study of fatigue crack initiation through characterizing DSGODs. In fact, the domain of data science and analytics has worked to formalize the signature discovery process in a number of different areas including cancer diagnostics, chemical forensics, and computer forensics $[13,31]$. Baker et al divided the signature discovery process into five main steps: specify the problem, inventory observables, specify measurements, assess and explore data, and develop the signature. In our study, we relied on a crack initiation theory to define our problem as identifying grains with intense localizations which could be precursors to crack initiation, our observables were governed by our specific experimental measurements, and by assessing a commonality of orientation information between these measurements, an indirect signature of CIP could be developed by quantifying intragrain lattice rotation through DSGOD statistics. Through this discovery process, other problems, specifically materials problems, can be studied beyond what is directly observable through experimental measurements by identifying indirect signatures. Signatures, like this CIP signature, are needed to continue the advancement of fatigue crack initiation studies.

\section{Acknowledgements}

Funding for this work has been provided by the US Department of Energy, Materials Sciences and Engineering Division, Basic Energy Sciences under Grant No. DE-SC0018901 (Dr. John Vetrano, Program Manager). This work is based upon research conducted at the FAST Beamline at the Cornell High Energy Synchrotron Source (CHESS) which is supported by the National Science Foundation under award DMR-1332208. The authors would also like to acknowledge Jeff Williams and Damien Texier for providing the material, Valery Valle for the development of the H-DIC method, Toby Francis for the collection of the Tribeam dataset, and Professor Armand Beaudoin and Professor Darren Pagan for insightful discussions.

\section{Methods}

\section{Material}

A block of wrought Inconel-718 (nominal composition in wt\% $\mathrm{Ni}-0.56 \% \mathrm{Al}-17.31 \% \mathrm{Fe}-0.14 \% \mathrm{Co}-17.97 \% \mathrm{Cr}$

$-5.4 \% \mathrm{Nb}-\mathrm{Ta}-1.00 \% \mathrm{Ti}-0.023 \% \mathrm{C}-0.0062 \% \mathrm{~N})$ was subjected to a 30 minute annealing treatment at 1050 ${ }^{\circ} \mathrm{C}$ followed by water quenching. A two-step precipitation hardening treatment consisting of 8 hours exposure at $720{ }^{\circ} \mathrm{C}$ then 8 hours at $620^{\circ} \mathrm{C}$ ) was conducted to form hardening $\gamma^{\prime}$ and $\gamma^{\prime \prime}$ precipitates [32]. The material possesses very weak crystallographic texture and an average grain size of $62 \mu \mathrm{m}$. 


\section{Electron Microscopy Characterization and Tension Experiment}

A flat dogbone-shaped tensile sample of the Inconel-718 material with gauge section $0.7 \mathrm{~mm} \times 3 \mathrm{~mm}$ was cut by wire electrical discharge machining. The tensile sample was mechanically mirror polished using abrasive papers and diamond suspensions then chemo-mechanically polished using a suspension of $0.04 \mu \mathrm{m}$ colloidal silica particles. A monotonic tension test was performed at room temperature using a custom in-situ \pm 5000 N stage within a ThermoFisher Versa3D microscope. Macroscopic strain was measured in-situ using fiducial markers located at both ends of the gauge length. Scanning electron microscopy (SEM) images were acquired before and during loading. The tension test was interrupted at macroscopic plastic strain levels of $1.26 \%$ and high resolution digital image correlation (HR-DIC) measurements were conducted. More details of the experimental procedure can be found elsewhere [33]. Further digital image correlation was performed using the heaviside digital image correlation (H-DIC) method [34,35]. 2D-EBSD measurements were performed with an EDAX OIM-Hikari XM4 EBSD detector using a step size of $0.2 \mu \mathrm{m}$. Diffraction patterns were acquired using an accelerating voltage of $20 \mathrm{kV}$, a $2 \times 2$ binning, and a beam current of $0.2 n \mathrm{~A}$. A 2D-EBSD map was acquired with a step size of $0.7 \mu \mathrm{m}$ prior and after deformation at the surface of the sample. All EBSD patterns were indexed using the Dictionary Indexing method developed by DeGraef et al. [36].

3D microstructure data were acquired using Tribeam tomography [15] in a sub-region where 2D-EBSD and H-DIC measurements were performed [28]. The TriBeam combines a femtosecond laser with a ThermoFisher Versa3D microscope for sample serial sectioning inside the scanning electron microscope at sub-micron voxel resolution over cubic-micron-scaled volumes. EBSD data were collected on a series of 526 consecutively sectioned slices (each $1 \mu \mathrm{m}$ thick), using an acceleration voltage of $30 \mathrm{kV}$ and a $4 \times 4$ binning. An EBSD resolution of $1 \mu \mathrm{m}$ was used on each slice to produce cubic voxels. The $3 \mathrm{D}$ volume was reconstructed using the Bluequartz Dream.3D open source software [37]. Individual grains were segmented using a misorientation tolerance angle of $5^{\circ}$ and twin boundaries were defined with a $2^{\circ}$ tolerance on both the plane and direction. The Paraview [38] software was used for data visualization.

\section{ff-HEDM Characterization and Cyclic Experiment}

The sample for the ff-HEDM experiment was fabricated from the same Inconel-718 material using wire electric discharged machining and has a tapered geometry with a gauge section of $1.5 \mathrm{~mm} \times 1 \mathrm{~mm} \times 1 \mathrm{~mm}$. The ff-HEDM experiment was conducted at the Forming and Shaping Technology (FAST) Beamline at the Cornell High Energy Syncrotron Source (CHESS). A schematic of the sample and experiment is provided in Figure 6(a). The specialized RAMS2 load frame mechanically loaded the sample for a total of 2 fullyreversed tension-compression cycles [39]. Each cycle was displacement controlled to a fixed strain amplitude 
of $1 \%$, with macroscopic strain monitoring in the gauge section provided by digital image correlation and load monitoring provided by a load cell above the sample.

The ff-HEDM measurements were performed using a monochromatic X-ray box beam with an energy of $61.332 \mathrm{keV}$ and resulted in a total diffraction volume of $1 \mathrm{~mm} \times 1 \mathrm{~mm} \times 0.3 \mathrm{~mm}$. Two Dexela 2923 detectors, each with dimensions 3888 pixels $\times 3072$ pixels and effective pixel pitch of $74.8 \mu \mathrm{m}$, were positioned side-byside downstream of the sample. Sample-to-detector distance $(976 \mathrm{~mm})$ and tilt angles relative to the beam were calibrated for each detector using a $\mathrm{CeO}_{2}$ powder [21].

The ff-HEDM measurements were collected in-situ at pre-determined load steps during initial loading and around the hysteresis loop. Figure 6(b) presents the locations of ff-HEDM measurements on the macroscopic stress-strain curve. During initial loading, measurements were captured before deformation and immediately before macroscopic yield. For cycles 1 and 2, ff-HEDM measurements were captured at seven macroscopic strain amplitudes $[1 \%, 0 \%,-0.5 \%,-1 \%, 0 \%, 0.5 \%, 1 \%]$ labeled by the sequence $[1 C, 2 C, 3 C, 4 C / 1 T, 2 T$, $3 T, 4 T]$. Prior to each ff-HEDM measurement, the load on the sample was halted and reduced by $10 \%$ to move the macroscopic stress off the yield surface and minimize inelastic material evolution during the measurements.

During each measurements, the sample was rotated $360^{\circ}$ about the loading axis to gather as many scattered diffraction events as possible for each grain in the diffracting volume. The rotation was discretized into $0.25^{\circ}$ steps where a diffraction image was collected. The series of diffraction images at each load step were processed to index and fit each grain in the aggregate using the HEXRD software package [21]. The definition of the stress metrics listed in Figure 7 are given in Table 1. The single crystal elastic moduli used in our constitutive relation to calculate grain-averaged stresses from the grain-averaged elastic strains are as follows: $C_{11}=259.6 \mathrm{GPa}, C_{12}=179 \mathrm{GPa}, C_{44}=109.6 \mathrm{GPa}[40]$.

\begin{tabular}{ll}
\hline Metric & Definition \\
\hline$\sigma_{L D}$ & $\begin{array}{c}\text { Loading direction normal stress component in the sample coordinate system, } \\
\\
\sigma_{i j}^{\text {sample }}=R_{i k} \sigma_{k l}^{\text {crystal }} R_{l j}, \sigma_{i j}^{\text {crystal }}=C_{i j k l} \epsilon_{k l}^{\text {crystal }}\end{array}$ \\
\hline $\begin{array}{l}\text { von Mises Stress } \\
\text { Maximum Resolve Shear }\end{array}$ & $\sigma^{\text {vm }}=\sqrt{\frac{3}{2} \sigma^{\text {dev }}: \sigma^{\text {dev }}}$, where $\sigma_{k l}^{\text {dev }}=\sigma_{k l}^{\text {sample }}-\sigma_{i i}^{\text {sample }} / 3$ \\
\multicolumn{1}{c}{ Stress Magnitude } & with $m_{k}$ is the slip plane normal and $n_{l}$ is the slip direction \\
\hline
\end{tabular}

Table 1: Summary of stress metrics and definitions utilized in the ff-HEDM Grain 414 case study. 


\section{Virtual Diffraction Simulation of the TriBeam Microstructure}

To determine the accuracy of ff-HEDM DSGOD reconstruction method as well as validate the effectiveness of utilizing ff-HEDM DSGOD statistics as a signature of localized plastic deformation, a virtual diffractometer was utilized to model a synthetic ff-HEDM experiment with the TriBeam microstructure. A virtual diffractometer simulates a rotating crystal X-ray diffraction experiment by correlating the position and orientation of a virtual crystallite to the sample rotation and the detector position of all the diffraction events associated with that crystallite; accounting for crystal structure, instrument setup, and experimental geometry $[24,25,41,42]$. The correlation is computed through calculating the scattering vector that satisfies Bragg's Law,

$$
\lambda=2 d_{h k l} \sin \theta_{h k l},
$$

where $h k l$ are the Miller indices of the diffracting plane (no indicial notation), $\lambda$ is the wavelength of the incoming X-ray, $d_{h k l}$ is the spacing of lattices planes defined by the diffraction plane, and $\theta_{h k l}$ is the Bragg angle. More details of the virtual diffractometer can be found elsewhere [24,25].

The TriBeam dataset is well suited for virtual diffraction since each voxel captures a cubic-micron sized crystalline region of the sample, with a well defined spatial location and crystallographic orientation. All parameters of the model were chosen to match the ff-HEDM experiment with certain assumptions to mimic ideal experimental conditions. The sample-to-detector distance was $980 \mathrm{~mm}$, the X-ray beam was perfectly collimated with an energy of $61.332 \mathrm{keV}$, and the detector dimensions and pitch exactly matched the detector configuration with perfect alignment to the incoming X-ray beam. To simulate the gradients in diffraction event intensity profiles, each diffracting voxel was assigned the same weighted intensity value since each voxel shared equal volume. At each diffraction event, a Lorentzian point spread function was applied to simulate indirect X-ray detection [25]. To simulate experimental detector noise, each synthetic diffraction image was subject to random noise sampled from a Gaussian probability distribution. Due to the high macroscopic strain amplitude used to deform the sample sectioned using the TriBeam, the sample was broken into 75 $\mu \mathrm{m}$ tall diffraction layers to reduce the number of overlapping diffraction peaks. For each layer, the sample was rotated $360^{\circ}$ about the vertical axis $y$ with diffraction images captured at $0.25^{\circ}$ steps for a total of 1440 images in the series. After the image series for each layer was collected, the DSGODs for each grain in the volume were reconstructed using the ff-HEDM DSGOD reconstruction method.

\section{EBSD DSGOD Reconstruction}

The spatially-resolved orientation information of surface (2D-EBSD) and subsurface (TriBeam) EBSD measurements provide a straight-forward method for calculating DSGODs. The data reduction process of EBSD 
data rasterizes the surface or volume into an array where each array element represents a crystallite comprised of a measured position and orientation. Grains are segmented by clustering neighboring array elements that share similar orientations. Due to the discretized nature of the data reduction process, array elements within a grain may share the exact same orientation in these data. We can define a probability $p_{\text {ebsd }}$ associated with an orientation $\mathbf{r}^{(i)}$ parameterized in the Rodrigues form as

$$
p_{e b s d}\left(\mathbf{r}^{(i)}\right)=\frac{v_{e b s d}\left(\mathbf{r}^{(i)}\right)}{\sum_{j=1}^{M} v_{e b s d}\left(\mathbf{r}^{(j)}\right)},
$$

where $v_{\text {ebsd }}\left(\mathbf{r}^{(i)}\right)$ is the number of array elements within a grain with orientation $\mathbf{r}^{(i)}$ and $M$ is the total number of array elements within a grain. Calculating the probability for every orientation within a grain results in a normalized distribution $p_{e b s d}(\mathbf{r})$, where $\sum_{i=1}^{M} p_{e b s d}\left(\mathbf{r}^{(i)}\right)=1$, and is equivalent to a DSGOD defined by Long and Miller [14]. We note that the 2D-EBSD technique only measures information from the grain exteriors that impinge on the sample surface and doesn't capture orientation information from the entirety of the grains' volume. Therefore, DSGODs constructed from 2D-EBSD datasets are not approximation of the grains' entire orientation distributions, rather select samplings from the grains' orientation distributions. However, this fact does not obstruct the goal of identifying a signature of high CIP.

\section{ff-HEDM DSGOD Reconstruction}

In this study, the single grain orientation distribution reconstruction method for ff-HEDM developed by Long and Miller and Nygren et al. was chosen due to the simplicity and computational efficiency of the algorithm, while still maintaining accurate approximations [14,43]. After the indexing and fitting of each grain in the ff-HEDM data, further processing of each diffraction event, or peak, is necessary to reconstruct a DSGOD. Using grain averaged information, the DSGOD algorithm generates a grid of $N$ trial orientations around the average orientation of each grain bounded by some amount of misorientation dependent on the amount of diffraction peak spread in the experiment. A bound of $4^{\circ}$ was used in the ff-HEDM experiment. A virtual diffractometer, with the parameters of the experiment, calculates the predicted diffraction event for each trial orientation, given in Rodrigues form as $\mathbf{r}^{(i)}$.

The algorithm collects the intensities of each predicted diffraction event from $\eta-\omega$ maps $[21,42]$ and uses the intensities to assign a measure proportional to the volume of the grain with an orientation, $v_{h e d m}\left(\mathbf{r}^{(i)}\right)$. The value $v_{\text {hedm }}\left(\mathbf{r}^{(i)}\right)$ is calculated by finding the maximum intensity collected while maintaining a completeness above an applied completeness threshold. A set of completeness thresholds ranging 0.7-0.85 were used for this work, depending on the experimental parameters such as flux and upstream attenuation. We 
can define a probability associated with some orientation $\mathbf{r}^{(i)}$ as

$$
p_{\text {hedm }}\left(\mathbf{r}^{(i)}\right)=\frac{v_{\text {hedm }}\left(\mathbf{r}^{(i)}\right)}{\sum_{j=1}^{N} v_{\text {hedm }}\left(\mathbf{r}^{(j)}\right)},
$$

where $p_{\text {hedm }}\left(\mathbf{r}^{(i)}\right)$ is the probability of some orientation $\mathbf{r}^{(i)}$ being present within a grain. This processing results in a normalized distribution $p_{\text {hedm }}(\mathbf{r})$, where $\sum_{i=1}^{N} p_{\text {hedm }}\left(\mathbf{r}^{(i)}\right)=1$. Note that Equations (4) and (5) result in the same DSGOD representation.

\section{Higher Order Statistics of DSGOD}

DSGODs serve as discrete approximations of probability distributions associated with the orientations of single grains. Being closely related to probability distributions, DSGODs lend themselves to traditional statistical tools used for characterizing distributions. These tools have already been presented by Long and Miller, but a short description of each will be given here for context [14]. In the formulation of the statistics, we assume small amounts of intragrain misorientation to allow for simpler computations. The first-order moment of a DSGOD is the mean orientation vector which describes the average orientation of a grain, of which all other higher order statistics rely on. The mean orientation vector for a grain can be defined from a DSGOD as

$$
\overline{\mathbf{r}}=\frac{\sum_{i=1}^{N} p^{(i)} \mathbf{r}^{(i)}}{\left\|\sum_{j=1}^{N} p^{(j)} \mathbf{r}^{(j)}\right\|},
$$

where $N$ is the total number of orientations in the DSGOD and $p^{(i)}$ is the probability of the $i$-th orientation $\mathbf{r}^{(i)}$ in Rodrigues form. We note $p^{(i)}$ is representative of both $p_{\text {ebsd }}^{(i)}$ and $p_{\text {hedm }}^{(i)}$. Like traditional calculations of higher order moments, the orientations of a DSGOD must be centered at the origin of orientation space, which is accomplished by subtracting the mean orientation from the orientations of a DSGOD. The mean subtracted orientation vectors are defined as

$$
\mathbf{r}_{m}^{(i)}=\mathbf{r}^{(i)}-\overline{\mathbf{r}}
$$

The second-order moment of a distribution is the covariance that describes the spread or size of an a distribution [44]. The expression for the covariance tensor of a DSGOD is defined as

$$
\boldsymbol{\Sigma}=\frac{N}{N-1} \sum_{i=1}^{N} p^{(i)}\left(\mathbf{r}_{m}^{(i)} \otimes \mathbf{r}_{m}^{(i)}\right)
$$


The third-order moment of a distribution is the skewness which details the asymmetry of the distribution [44]. The skewness of a DSGOD is a third-rank tensor defined as

$$
\boldsymbol{\Gamma}=\|\boldsymbol{\Sigma}\|^{-3 / 2} \sum_{i=1}^{N} p^{(i)}\left(\mathbf{r}_{m}^{(i)} \otimes \mathbf{r}_{m}^{(i)} \otimes \mathbf{r}_{m}^{(i)}\right)
$$

The fourth-order moment of a distribution is the kurtosis which details the relative weight of the tails of the distribution $[44,45]$. The kurtosis of a DSGOD is a fourth-rank tensor defined as

$$
\mathbf{K}=\|\boldsymbol{\Sigma}\|^{-2} \sum_{i=1}^{N} p^{(i)}\left(\mathbf{r}_{m}^{(i)} \otimes \mathbf{r}_{m}^{(i)} \otimes \mathbf{r}_{m}^{(i)} \otimes \mathbf{r}_{m}^{(i)}\right)
$$

In both definitions of skewness and kurtosis, $\|\boldsymbol{\Sigma}\|$ is the magnitude of the covariance tensor of the DSGOD and acts to normalize the metrics across different distributions. These moment tensors are reduced to scalar tensor magnitudes to reduce the dimensionality of this study.

\section{References}

[1] Ralph I. Stephens, Ali Fatemi, Robert R. Stephens, and Henry O. Fuchs. Metal Fatigue in Engineering. John Wiley and Sons, 2001.

[2] Michael D. Sangid. The physics of fatigue crack initiation. International Journal of Fatigue, 57:58 - 72, 2013. Fatigue and Microstructure: A special issue on recent advances.

[3] George Ellwood Dieter and David J. Bacon. Mechanical metallurgy, volume 3. McGraw-hill New York, 1976.

[4] Colin R. Gagg and Peter R. Lewis. In-service fatigue failure of engineered products and structures-case study review. Engineering Failure Analysis, 16(6):1775-1793, 2009.

[5] Norman E. Dowling. Mechanical behavior of materials: engineering methods for deformation, fracture, and fatigue. Prentice Hall international, 1993.

[6] Patrick Villechaise, Jonathan Cormier, Thomas Billot, and José Mendez. Mechanical behaviour and damage processes of Udimet 720Li: influence of localized plasticity at grain boundaries. In 12th International Symposium on Superalloys, pages 15-24, 2012.

[7] Baptiste Larrouy, Patrick Villechaise, Jonathan Cormier, and Olivier Berteaux. Grain boundary-slip bands interactions: Impact on the fatigue crack initiation in a polycrystalline forged ni-based superalloy. Acta Materialia, 99:325 - 336, 2015. 
[8] J. C. Stinville, M. A. Charpagne, F. Bourdin, P. G. Callahan, Z. Chen, M. P. Echlin, D. Texier, J. Cormier, P. Villechaise, T. M. Pollock, and V. Valle. Measurement of elastic and rotation fields during irreversible deformation using heaviside-digital image correlation. Materials Characterization, 169:110600, 2020.

[9] J. C. Stinville, M. P. Echlin, D. Texier, F. Bridier, P. Bocher, and T. M. Pollock. Sub-grain scale digital image correlation by electron microscopy for polycrystalline materials during elastic and plastic deformation. Experimental Mechanics, 56(2):197-216, 2016.

[10] J. C. Stinville, P. G. Callahan, M. A. Charpagne, M. P. Echlin, V. Valle, and T. M. Pollock. Direct measurements of slip irreversibility in a nickel-based superalloy using high resolution digital image correlation. Acta Materialia, 186:172 - 189, 2020.

[11] Zhenbo Zhang, Zhibiao Yang, Song Lu, Allan Harte, Roberto Morana, and Michael Preuss. Strain localisation and failure at twin-boundary complexions in nickel-based superalloys. Nature Communications, 11(1):4890, Sep 2020.

[12] Stuart I. Wright, Seiichi Suzuki, and Matthew M. Nowell. In situ ebsd observations of the evolution in crystallographic orientation with deformation. JOM, 68(11):2730-2736, 2016.

[13] Nathan A. Baker, Jonathan L. Barr, George T. Bonheyo, Cliff A. Joslyn, Kannan Krishnaswami, Mark E. Oxley, Rich Quadrel, Landon H. Sego, Mark F. Tardiff, and Adam S. Wynne. Research towards a systematic signature discovery process. In 2013 IEEE International Conference on Intelligence and Security Informatics, pages 301-308, 2013.

[14] Timothy J. H. Long and Matthew P. Miller. Statistical characterization of intragrain misorientations at large strains using high-energy x-ray diffraction: Application to hydrogen embrittlement. Integrating Materials and Manufacturing Innovation, 8(3):423-439, 2019.

[15] McLean P. Echlin, Marcus Straw, Steven Randolph, Jorge Filevich, and Tresa M. Pollock. The tribeam system: Femtosecond laser ablation in situ sem. Materials Characterization, 100:1 - 12, 2015.

[16] L. Margulies, G. Winther, and H. F. Poulsen. In situ measurement of grain rotation during deformation of polycrystals. Science, 291(5512):2392-2394, 2001.

[17] H. F. Poulsen, L. Margulies, S. Schmidt, and G. Winther. Lattice rotations of individual bulk grains: Part i: 3d x-ray characterization. Acta Materialia, 51(13):3821 - 3830, 2003. 
[18] H. F. Poulsen, U. Lienert, and W. Pantleon. Characterisation of orientation distributions of individual grains within deformed metals. Materials Science and Technology, 21(12):1397-1400, 2005.

[19] R. M. Suter, D. Hennessy, C. Xiao, and U. Lienert. Forward modeling method for microstructure reconstruction using x-ray diffraction microscopy: Single-crystal verification. Review of Scientific Instruments, 77(12):123905, 2006.

[20] M. P. Miller, J.-S. Park, P. R. Dawson, and T.-S. Han. Measuring and modeling distributions of stress state in deforming polycrystals. Acta Materialia, 56(15):3927 - 3939, 2008.

[21] Joel Bernier, Nathan Barton, U. Lienert, and M. Miller. Far-field high-energy diffraction microscopy: A tool for intergranular orientation and strain analysis. The Journal of Strain Analysis for Engineering Design, 46:527-547, 102011.

[22] Diwakar Naragani, Michael D. Sangid, Paul A. Shade, Jay C. Schuren, Hemant Sharma, Jun-Sang Park, Peter Kenesei, Joel V. Bernier, Todd J. Turner, and Iain Parr. Investigation of fatigue crack initiation from a non-metallic inclusion via high energy x-ray diffraction microscopy. Acta Materialia, 137:71 84, 2017.

[23] Sven Gustafson, Wolfgang Ludwig, Paul Shade, Diwakar Naragani, Darren Pagan, Phil Cook, Can Yildirim, Carsten Detlefs, and Michael D Sangid. Quantifying microscale drivers for fatigue failure via coupled synchrotron x-ray characterization and simulations. Nature Communications, 11(1):3189, June 2020.

[24] W. R. Busing and H. A. Levy. Angle calculations for 3- and 4-circle X-ray and neutron diffractometers. Acta Crystallographica, 22(4):457-464, Apr 1967.

[25] Su Leen Wong, Jun-Sang Park, M. P. Miller, and P. R. Dawson. A framework for generating synthetic diffraction images from deforming polycrystals using crystal-based finite element formulations. Computational Materials Science, 77:456 - 66, 2013.

[26] Charles Frank. Orientation mapping. Metallurgical Transactions A, 13(3):403-408, Mar 1988.

[27] Stuart P. Lloyd. Least squares quantization in pcm. IEEE Transactions on Information Theory, 28:129$137,1982$.

[28] M. A. Charpagne, J. C. Stinville, P. G. Callahan, D. Texier, Z. Chen, P. Villechaise, V. Valle, and T. M. Pollock. Automated and quantitative analysis of plastic strain localization via multi-modal data recombination. Materials Characterization, 163:110245, 2020. 
[29] Matthew P. Miller, Darren C. Pagan, Armand J. Beaudoin, Kelly E. Nygren, and Dalton J. Shadle. Understanding micromechanical material behavior using synchrotron x-rays and in situ loading. Metallurgical and Materials Transactions A, 51(9):4360-4376, Sep 2020.

[30] Nathan R. Barton and Joel V. Bernier. A method for intragranular orientation and lattice strain distribution determination. Journal of Applied Crystallography, 45(6):1145-1155, 2012.

[31] Sheriff Jolaoso, Russ Burtner, and Alex Endert. Toward a deeper understanding of data analysis, sensemaking, and signature discovery. In Human-Computer Interaction - INTERACT 2015, pages 463-478. Springer International Publishing, 2015.

[32] Damien Texier, Ana Casanova Gómez, Stéphane Pierret, Jean-Michel Franchet, Tresa M. Pollock, Patrick Villechaise, and Jonathan Cormier. Microstructural features controlling the variability in lowcycle fatigue properties of alloy inconel 718da at intermediate temperature. Metallurgical and Materials Transactions A, 47(3):1096-1109, January 2016.

[33] J. C. Stinville, M. P. Echlin, D. Texier, F. Bridier, P. Bocher, and T. M. Pollock. Sub-grain scale digital image correlation by electron microscopy for polycrystalline materials during elastic and plastic deformation. Experimental Mechanics, pages 1-20, 2015.

[34] F. Bourdin, J. C. Stinville, M. P. Echlin, P. G. Callahan, W. C. Lenthe, C. J. Torbet, D. Texier, F. Bridier, J. Cormier, P. Villechaise, T. M. Pollock, and V. Valle. Measurements of plastic localization by heaviside-digital image correlation. Acta Materialia, 157:307-325, 2018.

[35] Valéry Valle, Stephen Hedan, Philippe Cosenza, Anne-Laure Fauchille, and M. Berdjane. Digital image correlation development for the study of materials including multiple crossing cracks. Experimental Mechanics, 55(2):379-391, 2015.

[36] M. A. Jackson, E. Pascal, and M. De Graef. Dictionary indexing of electron back-scatter diffraction patterns: a hands-on tutorial. Integrating Materials and Manufacturing Innovation, 8(2):226-246, May 2019.

[37] Michael A. Groeber and Michael A. Jackson. DREAM.3d: A digital representation environment for the analysis of microstructure in 3d. Integrating Materials and Manufacturing Innovation, 3(1):56-72, April 2014.

[38] James Ahrens, Berk Geveci, and Charles Law. Paraview: An end-user tool for large data visualization. The Visualization Handbook, 717, 2005. 
[39] Paul A. Shade, Basil Blank, Jay C. Schuren, Todd J. Turner, Peter Kenesei, Kurt Goetze, Robert M. Suter, Joel V. Bernier, Shiu Fai Li, Jonathan Lind, Ulrich Lienert, and Jonathan Almer. A rotational and axial motion system load frame insert for in situ high energy x-ray studies. Review of Scientific Instruments, 86(9):093902, 2015.

[40] G. Martin, N. Ochoa, K. Saï, E. Hervé-Luanco, and G. Cailletaud. A multiscale model for the elastoviscoplastic behavior of directionally solidified alloys: Application to fe structural computations. International Journal of Solids and Structures, 51(5):1175-1187, 2014.

[41] Darren C. Pagan and Matthew P. Miller. Connecting heterogeneous single slip to diffraction peak evolution in high-energy monochromatic X-ray experiments. Journal of Applied Crystallography, 47(3):887898, Jun 2014.

[42] Kelly E. Nygren, Darren C. Pagan, Joel V. Bernier, and Matthew P. Miller. An algorithm for resolving intragranular orientation fields using coupled far-field and near-field high energy x-ray diffraction microscopy. Materials Characterization, 165:110366, 2020.

[43] K. E. Nygren, D. C. Pagan, and M. P. Miller. Sub-grain orientation resolution during continuous loading using only far-field HEDM. IOP Conference Series: Materials Science and Engineering, 580:012018, December 2019 .

[44] H. Cramer. The elements of probability theory. Quarterly Journal of the Royal Meteorological Society, 81(350):649-649, 1955.

[45] Peter H. Westfall. Kurtosis as peakedness. The American Statistician, 68(3):191-195, 2014. 